\title{
Adjuvant and Neo-Adjuvant Therapy for Gastric Cancer: The Standard By Geographic Location
}

\author{
Shouki Bazarbashi \\ King Faisal Specialist Hospital and Research Center, Riyadh, Saudi Arabia
}

Corresponding Author: Shouki Bazarbashi

\author{
E-mail: Bazarbashi@gmail.com
}

A total of 989,600 new stomach cancer cases and 738,000 deaths are estimated to have occurred wolrdwide in 2008 , accounting for $8 \%$ of the total cancer cases worldwide and $10 \%$ of total Deaths ${ }^{1}$. The highest incidence rates are in Eastern Asia, Eastern Europe, and South America and the lowest rates are in North America and most parts of Africa. Stomach cancer rates have decreased substantially in most parts of the world, in part due to factors related to the increased use and availability of refrigeration including the increased availability of fresh fruits and vegetables, and a decreased reliance on salted and preserved foods. Other major determinants for the favorable trends are reductions in chronic $\mathrm{H}$. pylori infection in most parts of the world and smoking in some parts of the developed world ${ }^{1}$.

The prognosis of gastric cancer is quite poor. Despite progress in the development of new management strategies, surgery is still the only curative treatment. Unfortunately, gastric cancer is difficult to diagnose at an early stage. Using the International Union Against Cancer (UICC) and American Joint Committee on Cancer (AJCC) staging system ${ }^{2}$, nearly 65 per cent of patients in the USA are initially diagnosed at an advanced stage (T3/T4), and 85 per cent of them have lymph node metastasis ${ }^{3}$. The mean survival time of these patients is 24 months and the 5-year survival rate is only $20-40$ per cent after surgery ${ }^{4}$.

This poor results have driven investigators to use other form of therapy, namely chemotherapy and radiation in addition to surgery to improve survival. Over the past several years, 3 standards of care for adjuvant and neoadjuvant therapy for gastric cancer have emerged in different parts of the worlds. Those include adjuvant chemoradiotherapy using 5-fluorouracil (FU) and leucovorin as per the intergroup trial ${ }^{5}$, adopted in the USA, perioperative chemotherapy with the ECF (epirubicin, cisplatin and FU) regimen as per the Magic trial, adopted in Europe $^{6}$ and adjuvant chemotherapy with either S1 or Xelox regimen (Capecitabine and Oxaliplatin) adopted in east Asia ${ }^{7,8}$.

\section{The US experience:}

The largest and most recent trial, US Intergroup INT0116, provides the most compelling data in support of adjuvant chemoradiotherapy following complete surgical resection, particularly since it used contemporary radiotherapy (RT) techniques and concurrently administered fluoropyrimidine radiosensitization ${ }^{5}$. Following potentially curative resection of gastric $(79 \%)$ or esophagogastric junction (EGJ) cancer (21\%) T1-4, N0-1), 556 patients were randomly assigned to observation alone or adjuvant combined chemoradiotherapy. Treatment consisted of one cycle of FU (425 mg/m² per day) and leucovorin calcium $\left(20 \mathrm{mg} / \mathrm{m}^{2}\right.$ per day) daily for five days, followed one month later by External beam radiotherapy (RT) (45 Gy in daily $1.8 \mathrm{~Gy}$ fractions) given with concurrent $\mathrm{FU}$ and leucovorin calcium ( $400 \mathrm{mg} / \mathrm{m}^{2}$ and $20 \mathrm{mg} / \mathrm{m}^{2}$, respectively) on days 1 through 4 , and on the last three days of RT. Two more five-day cycles of chemotherapy (FU $425 \mathrm{mg} / \mathrm{m}^{2}$ per day and leucovorin calcium $20 \mathrm{mg} / \mathrm{m}^{2}$ per day) were given at monthly intervals beginning one month after completion of chemoradiotherapy.

The majority of tumors were T3/T4 (68 and 69 percent of the treated and control groups, respectively), and 85 percent had nodal metastases. Three-year disease-free (48 versus 31 percent) and overall survival rates (50 versus 41 percent) were significantly better with combined modality therapy, and median survival was significantly longer (36 versus 27 months). Benefits were maintained with longer followup (five-year overall survival 43 versus 28 percent, hazard ratio [HR] for survival 1.32 (95\% CI $1.10-1.60)^{9}$. In the chemoradiotherapy group, grade 3 and grade 4 acute toxic effects occurred in 41 and 32 percent of patients, respectively, while three (1 percent) died from treatment-related toxicity

The initial report of this study changed the standard of care in the US following potentially curative resection 
of gastric cancer from observation alone to surgery followed by adjuvant combined chemoradiotherapy. A criticism of this trial was the limited extent of the surgical procedure in most cases. Although D2 lymph node dissection (removal of nodes along the hepatic, left gastric, celiac and splenic arteries, and in the splenic hilum) was recommended, it was only performed in 10 percent of cases, and 54 percent did not even have clearance of the D1 (perigastric) nodal regions. This noncompliance likely contributed to inferior survival and a 64 percent relapse rate in the surgery alone arm.

\section{The European experience:}

Two large positive trials and one negative Dutch trial $^{10}$ established peri-operative chemotherapy as the standard of care for resectable gastric or EGJ cancer.

The largest and most influential trial is the United Kingdom Medical Research Council MAGIC trial ${ }^{6}$, in which 503 patients with potentially resectable gastric (74 percent), distal esophageal (11 percent), or EGJ adenocarcinomas (15 percent) were randomly assigned to surgery alone or surgery plus perioperative chemotherapy(threepreoperativeand threepostoperative cycles of epirubicin, cisplatin and infusional FU $[E C F])$. A higher proportion of chemotherapy-treated patients with gastric cancer who underwent radical surgery had a potentially curative procedure ( 79 versus 70 percent), and significantly more had $\mathrm{T} 1 / 2$ tumors (52 versus 37 percent) and N0/N1 disease ( 84 versus 71 percent). Chemotherapy was well tolerated overall; excluding patients with neutropenia (23 percent), fewer than 12 percent of all patients had serious (grade 3 or 4) toxic effects. However, only 104 (42 percent) were able to complete protocol treatment, including surgery and all three cycles of the postoperative chemotherapy. These data underscore one of the major problems with the perioperative approach, which is the difficulty in administering the full number of postoperative chemotherapy cycles. Overall survival was significantly better in the chemotherapy group (hazard ratio [HR] for death $0.75,95 \%$ CI $0.60-0.93$ ) as was progression-free survival (PFS, HR for progression 0.66). The 25 percent reduction in the risk of death favoring chemotherapy translated into an improvement in five-year survival from 23 to 36 percent. This trial was criticized for poor staging procedures (no endoscopic ultrasound and no peritoneal washing).

A similar benefit for perioperative chemotherapy was noted in a French multicenter trial in which 224 patients with potentially resectable stage II or greater adenocarcinoma of the stomach $(\mathrm{n}=55)$, EGJ $(\mathrm{n}=144)$ or distal esophagus $(n=25)$ were randomly assigned to two to three cycles of preoperative chemotherapy (infusional FU $800 \mathrm{mg} / \mathrm{m}^{2}$ daily for five days plus cisplatin $100 \mathrm{mg} / \mathrm{m}^{2}$ on day 1 or 2 , every four weeks) or surgery alone ${ }^{11}$ ). Patients in the chemotherapy arm were to receive three to four cycles of postoperative chemotherapy as well.

Patients undergoing neoadjuvant chemotherapy were significantly more likely to undergo R0 (microscopically complete) resection (84 versus 73 percent), and there was a statistically insignificant trend toward fewer node-positive tumors (67 versus 80 percent, $P=0.054$ ) that favored this group as well. Among the patients who received at least one cycle of preoperative chemotherapy, only one-half received any postoperative chemotherapy. With a median 5.7-year follow-up, perioperative chemotherapy was associated with a significant 35 percent reduction in the risk of disease recurrence (five-year disease-free survival 34 versus 19 percent) and a significant, 31 percent lower risk of death (five-year survival 38 versus 24 percent).

\section{The East Asian experience:}

More than 30 randomized trials have compared adjuvant systemic chemotherapy to surgery alone in resectable gastric cancer, with variable, mostly negative results when overall survival is considered as the primary endpoint. It is clear that some of the trials were underpowered to detect a significant survival difference.

Given the variability in outcomes in these phase III trials, several meta-analyses have been undertaken, all of which support a significant survival benefit for adjuvant chemotherapy Table 1 . Several studies indicate a somewhat better prognosis in Asian as compared to Western populations with gastric cancer ${ }^{12-16}$.

Table 1. Meta-analysis of adjuvant chemotherapy in resected gastric cancer

\begin{tabular}{lccc}
\hline Author & $\begin{array}{c}\text { Number } \\
\text { of } \\
\text { studies }\end{array}$ & $\begin{array}{c}\text { Number } \\
\text { of } \\
\text { patients }\end{array}$ & $\begin{array}{c}\text { Odds ratio/hazard } \\
\text { ratio for death } \\
(\mathbf{9 5 \% C I})\end{array}$ \\
\hline Hermans, et al. ${ }^{12}$ & 11 & 2096 & $0.88(0.78-1.08)$ \\
\hline Earle, et al. ${ }^{13}$ & 13 & 1990 & $0.80(0.66-0.97)^{*}$ \\
\hline Mari, et al. ${ }^{14}$ & 20 & 3658 & $0.82(0.75-0.89)^{*}$ \\
\hline $\begin{array}{l}\text { Januger, } \text { et al. }{ }^{15} \\
\text { Sun, et al. }{ }^{16}\end{array}$ & 21 & 3962 & $0.84(0.74-0.96)^{*}$ \\
\hline Paoletti, et al. ${ }^{17}$ & 12 & 3809 & $0.78(0.71-0.85)^{*}$ \\
\hline
\end{tabular}


The most recent of these analyses evaluated individual patient data from 17 randomized trials comparing adjuvant systemic chemotherapy versus surgery alone, conducted in both Asian and Western populations (but not including the largest Japanese trial evaluating adjuvant $\mathrm{S}-1$ ), and which required complete (R0) resection prior to enrollment ${ }^{17}$. The risk of death in patients receiving an adjuvant chemotherapy regimen that contained a fluoropyrimidine was reduced by 18 percent (HR for death $0.82,95 \%$ CI 0.75 to 0.90$)$.

The benefit of adjuvant treatment with S-1 was demonstrated in a Japanese ACTS-GC trial in which 1059 patients with stage II or III gastric cancer who had undergone potentially curative surgery with D2 lymphadenectomy were randomly assigned to six months of S1 (80 to $120 \mathrm{mg}$ daily for four weeks, repeated every six weeks for one year) versus surgery alone ${ }^{7}$. Fiveyear overall survival was significantly better with S-1 (72 versus 61 percent), with better than expected survival in both groups. By comparison, the five-year survival rates for the INT0116 and the MAGIC trials were 43 versus 28 percent, and 36 versus 23 percent for the treatment and control groups, respectively.

These results led to the adoption of one year of postoperative S-1 as a standard adjuvant therapy approach for gastric cancer in East Asian patients. It is difficult to know whether the benefit of adjuvant therapy with S-1, as demonstrated in the ACTS-GC trial, can be extrapolated to other populations, given the markedly better outcomes seen in both the treated and the surgery alone control groups, stage for stage, when compared to outcomes in other non-Japanese populations.S-1 is approved in Japan for adjuvant therapy of gastric cancer and in Europe for treatment of advanced gastric cancer; it is not available in the US.

The benefit of adjuvant therapy using capecitabine in combination with oxaliplatin was addressed in the multicenter CLASSIC trial, in which 1035 patients with stage II, IIIA, or IIIB gastric cancer were randomly assigned to eight cycles of capecitabine $\left(1000 \mathrm{mg} / \mathrm{m}^{2}\right.$ twice daily in days 1 to 14$)$ plus oxaliplatin $(130 \mathrm{mg} /$ $\mathrm{m}^{2}$ on day 1) or surgery alone after D2 gastrectomy ${ }^{8}$. The study was conducted in South Korea, China, and Taiwan. Only 567 percent of the patients assigned to chemotherapy received all eight cycles of chemotherapy as planned, and adverse events (most commonly neutropenia, nausea, vomiting, thrombocytopenia, and anorexia) led to chemotherapy dose modifications in 90 percent of patients. Despite this, at a median followup of 34 months, chemotherapy was associated with a significant improvement in three-year disease-free survival (74 versus 59 percent, HR for death 0.56 , $95 \%$ CI $0.44-0.72$ ), with only a borderline statistically significant improvement in overall survival (83 versus 78 percent, HR $0.72,95 \%$ CI $0.52-1.00)$.

\section{Which approach is superior?}

Several studies has been performed to establish the superiority of one approach over the other. The following is a suppury of thses trials.

Adjuvant chemoradiotherapy vs chemotherapy alone. In the largest trial, the ARTIST trial, 458 patients with complete resected gastric cancer and a D2 lymph node dissection were randomly assigned to six courses of postoperative capecitabine plus cisplatin (XP) or two courses of postoperative XP followed by chemoradiotherapy (45 Gy RT with concurrent daily capecitabine [ $825 \mathrm{mg} / \mathrm{m}^{2}$ twice daily]) and two additional courses of $\mathrm{XP}^{18}$. Compared to chemotherapy alone, the addition of RT to XP chemotherapy did not significantly reduce recurrence rates, although in unplanned subgroup analysis, patients with nodal metastases had superior disease-free survival with combined therapy as compared to XP alone. Overall survival, a secondary endpoint, was not analyzed. At a median follow-up of 53 months, estimated three-year DFS (the primary endpoint) was not significantly better in patients who received combined modality therapy (78 versus 74 percent, $P=0.0862)$, although unplanned subset analysis did indicate a significantly better outcome with chemoradiotherapy in those with node-positive disease. The hypothesis that adjuvant chemoradiotherapy may represent a better approach than adjuvant chemotherapy for patients with node-positive disease will be tested in a successor trial, the ARTIST-II trial.

A Greek trial randomized patients with histologically proven,radically resected gastric cancer, stage , T3 and/or $\mathrm{N}+$ to 6 cycles of adjuvant docetaxel with cisplatin, both at $75 \mathrm{mg} / \mathrm{m}^{2}$ every 3 weeks ( $\operatorname{arm~A}$ ) or the same treatment with RT (arm B; 45 Gy). A total of 147 patients were randomized. After a median follow-up of 53.7 months, no differences in overall (OS) and disease free survival (DFS) were found between the two arms. The author concluded that the addition of RT to platinum/docetaxel adjuvant chemotherapy does not appear to improve survival in high-risk, radically resected gastric cancer ${ }^{19}$.

The only trial to show a significant survival benefit for the addition of RT randomly assigned 68 patients undergoing complete resection with a D1 or D2 lymph node dissection for previously untreated gastric cancer to chemoradiotherapy administered according to the INT 0116 trial (but using intensity modulated RT) or chemotherapy alone (five cycles of FU $425 \mathrm{mg} / \mathrm{m}^{2}$ per day and leucovorin calcium $25 \mathrm{mg} / \mathrm{m}^{2}$ per day given five days in a row, once monthly $)^{20}$. All patients were followed for at least three years. Three-year disease-free survival 
Kasr-El-Aini Journal Of Clinical Oncology And Nuclear Medicine

Vol. 9|No. 1-2 2013

Shouki Bazarbashi

rate was significantly higher in the chemoradiotherapy group (56 versus 29 percent) as was overall survival (68 versus 44 percent).

Pre-operative chemotherapy vs post-operative chemotherapy: the SAKK trial attempted to address the issue of pre-operative vs post-operative DCF(docetaxel, cisplatin and fluorouracil). Unfortunately the trial was closed pre-maturely because of poor patient accrual, and the question remains unanswered ${ }^{21}$.

Peri-operative chemotherapy vs pre-operative chemotherapy with post-operative chemoradiotherapy: this issue is being address by the CRITICS trial. Results of this study will demonstrate whether the combination of preoperative chemotherapy and postoperative chemoradiotherapy will improve the clinical outcome of the current European standard of perioperative chemotherapy, and will therefore play a key role in the future management of patients with resectable gastric cancer $^{22}$.

Attempts to improve current therapies: several trials have attempted to improve on the current results by either intensifying the chemotherapy, adding targeted agents or utilizing intraperitoneal approach.

AMC 0201 trial examined the benefit of intensifying adjuvant $\mathrm{Mf}$ (mitomycin $\mathrm{C}$ and FUDR) chemotherapy, by prolonging the administration of oral fluoropyrimidine (F) and added cisplatin (P) to Mf (MFP) and compared it to the standard MF regimen in a phase III randomized trial. It aimed to determine whether this strategy could improve the 3-year relapse free survival (RFS) in curatively resected gastric cancer patients ${ }^{23}$. With a median follow up of $6.6 \mathrm{yrs}$, there was no difference in RFS between the two groups (HR, 1.10; 95\% C.I. $0.89-$ $1.35 ; P=0.3918$; 5 yRFS $61.1 \%$ in Mf and $57.9 \%$ in MFP). Difference in overall survival (OS) was also insignificant (HR, 1.11; 95\% C.I. $0.89-1.39 ; P=0.3349 ; 5$ yOS $66.5 \%$ in Mf and $65.0 \%$ in MFP).

The CALBG 80101 trial examined the benefit of using a potentially more active regimen (ECF) instead of 5FU and leucovorin used in the INT 0116 tial in the context of adjuvant chemoradiotherapy ${ }^{24}$. 546 patients were enrolled. The result showed that following curative resection of gastric or GEJ adenocarcinoma, postoperative chemoradiotherapy using ECF before and after 5-FU/RT does not improve survival when compared to bolus 5-FU/ Leucovorin (LV) before and after 5-FU/RT. Median OS was 37 months in the 5FU/LV arm and 38 months in the ECF arm (HR, 1.03; 95\% CI, 0.80-1.34; $P=0.80)$.

MAGI_B trial: In the era of targeted therapy, biologic agents have also been investigated for adjuvant treatment of gastric cancer. Based on the success of the MAGIC trial in the United Kingdom, MAGIC-B trial (MRC-ST03) is ongoing to determine the efficacy of adding bevacizumab to adjuvant therapy (perioperative epirubicin, capecitabine, and cisplatin with or without bevacizumab).

AMC 0101 trial: In an attempt to improve the postoperative adjuvant chemotherapy in serosa-involving advanced gastric cancer, Kang and his colleagues from South Korea adopted the strategies of intraperitoneal and early start of chemotherapy, as well as prolongation of oral fluoropyrimidine and addition of cisplatin (P) to $\mathrm{Mf}$ (mitomycin and doxifluridine) regimen in the AMC 0101 trial $^{25}$. Patients whose tumor were grossly seroa-positive and able to be resected curatively were randomized during operation to receive either Mf or iceMFP. Patients with postoperative pathologic stage I or IV (M1) were excluded after surgery. For Mf, $20 \mathrm{mg} / \mathrm{m}^{2}$ of mitomycin-C (M) was injected 3-6 weeks after surgery and 4 wks later, $460-600 \mathrm{mg} / \mathrm{m}^{2} /$ day of doxifluridine was administered orally for 3 months. For iceMFP, $100 \mathrm{mg}$ of $\mathrm{P}$ in $1 \mathrm{~L}$ of saline was administered intraperitoneally for $2 \mathrm{~h}$ during surgery and $15 \mathrm{mg} / \mathrm{m}^{2}$ of $\mathrm{M}$ was injected 1 day after surgery. Doxifluridine was started 4 wks after surgery and extended for a total of 12 months and 6 shots of monthly $60 \mathrm{mg} / \mathrm{m}^{2}$ of $P$ were added. In an updated analysis as of April 2011, with a median follow-up of 6.6 years, iceMFP group had a significantly longer RFS (HR, 0.73; 95\% C.I. $0.57-0.93 ; P=0.0092$ ) and overall survival (HR, $0.77 ; 95 \%$ C.I. $0.60-0.98 ; P=0.0365$ ) than Mf group.

\section{Is gastric cancer one disease?}

Gastric cancer has been considered a single heterogenous disease with several epidemiologic and histopathologic characteristics; for the purposes of medical management, gastric cancer is treated in a uniform fashion, without regard to subtype. Pathologically, gastric adenocarcinoma may be distinguished according to the Lauren's classification as intestinal, diffuse, or mixed subtypes $^{26}$. Epidemiologically, intestinal gastric cancer, particularly of the antrum, is strongly associated with chronic inflammation (i.e., atrophic gastritis) $)^{27,28}$ often as a consequence of chronic infection with Helicobacter pylori $^{29,30}$. Conversely, inflammation is characteristically absent in the development of Lauren's diffuse type gastric cancer, particularly when as a result of a germline mutation in $\mathrm{CDH}^{31}$. Anatomically, proximal gastric cancer may be classified as a third type of gastric cancer, as tumors of the gastric cardia/gastroesophageal junction (GEJ), for which inflammation of a different type (i.e., chronic gastric acid/ bile reflux) may be the driving force for carcinogenesis ${ }^{32,33}$. Proximal/GEJ tumors are also usually not diffuse in histology, similar to distal nondiffuse gastric cancer. Shah and his colleagues found that individual diseases arising from the stomach defined a priori, namely "proximal 
nondiffuse," "diffuse," and "distal nondiffuse" gastric cancer, have distinct gene expression ${ }^{34}$. Their analysis suggests that (i) different types of cancers arise from the stomach, (ii) there likely exist unique molecular drivers that may be identified among specific genetic pathways that distinguish each disease, and (iii) the presence of different biomarkers and therapeutic targets for each disease is also likely. Accordingly new trials are needed to assess each adjuvant/neo-adjuvant approach on the basis of the new molecular classification in order to examine the efficacy of each approach separately.

\section{CONCLUSION}

In conclusion, several approaches have been established to improve survival in patient with resectable gastric cancer. At present, unfortunately each of those approaches is used largely depending on the geographic location rather than superiority over the other. Moreover, none of the above mentioned approaches has been established as effective in one genetic subtype rather than the other. New trials should be stratified in addition to the new genetic subtypes.

\section{REFERENCES}

1. Jemal A, Bray F, Center MM, Ferlay J, Ward E, Forman D. Global cancer statistics. CA Cancer J.Clin. 2011;61(2):69-90.

2. Dicken BJ, Bigam DL, Cass C, Mackey JR, Joy AA, Hamilton SM. Gastric adenocarcinoma: Review and considerations for future directions. Ann.Surg. 2005;241(1):27-39.

3. Hundahl SA, Phillips JL, Menck HR. he National Cancer Data Base report on poor survival of U.S. gastric carcinoma patients treated with gastrectomy: Fifth Edition American Joint Committee on Cancer Staging, Proximal Disease and the 'Different Disease' Hypothesis. Cancer 2000;88(4):921-32.

4. Hartgrink HH, Putter $\mathrm{H}$, Klein Kranenbarg E, Bonenkamp JJ, van de Velde CJ. Value of palliative resection in gastric cancer. Br.J.Surg. 2002;89(11):1438-43.

5. Macdonald JS, Smalley SR, Benedetti J, Hundahl SA, Estes NC, Stemmermann GN, et al. Chemoradiotherapy after surgery compared with surgery alone for adenocarcinoma of the stomach or gastroesophageal junction. N.Engl.J.Med. 2001;345(10):725-30.

6. Cunningham D, Allum WH, Stenning SP, Thompson JN, Van De Velde CJH, Nicolson M, et al. Perioperative chemotherapy versus surgery alone for resectable gastroesophageal cancer. N.Engl.J.Med. 2006;355(1):11-20.

7. Sasako M, Sakuramoto S, Katai H, Kinoshita T, Furukawa H, Yamaguchi T, et al. Five-year outcomes of a randomized phase III trial comparing adjuvant chemotherapy with S-1 versus surgery alone in stage II or III gastric cancer. J.Clin.Oncol. 2011;29(33):4387-93.
8. Bang YJ, Kim YW, Yang HK, Chung HC, Park YK, Lee $\mathrm{KH}$, et al. Adjuvant capecitabine and oxaliplatin for gastric cancer after D2 gastrectomy (CLASSIC): A phase 3 open-label, randomised controlled trial. Lancet 2012;379(9813):315-21.

9. Smalley SR, Benedetti JK, Haller DG, Hundahl SA, Estes NC, Ajani JA, et al. Updated analysis of SWOGdirected intergroup study 0116: A phase III trial of adjuvant radiochemotherapy versus observation after curative gastric cancer resection. J.Clin.Oncol. 2012; 30(19):2327-33.

10. Songun I, Keizer HJ, Hermans J, Klementschitsch P, De Vries JE, Wils JA, et al. Chemotherapy for operable gastric cancer: Results of the Dutch randomised FAMTX trial. Eur.J.Cancer 1999;35(4):558-62.

11. Ychou M, Boige V, Pignon JP, Conroy T, Bouché O, Lebreton $\mathrm{G}$, et al. Perioperative chemotherapy compared with surgery alone for resectable gastroesophageal adenocarcinoma: An FNCLCC and FFCD multicenter phase III trial. J.Clin.Oncol. 2011;29(13):1715-21.

12. Hermans J, Bonenkamp JJ, Boon MC, Bunt AMG, Ohyama S, Sasako M, et al. Adjuvant therapy after curative resection for gastric cancer: Meta-analysis of randomized trials. J.Clin.Oncol. 1993;11(8):1441-7.

13. Earle CC, Maroun JA. Adjuvant chemotherapy after curative resection for gastric cancer in non-asian patients: Revisiting a meta-analysis of randomised trials. Eur.J.Cancer 1999;35(7):1059-64.

14. Mari E, Floriani I, Tinazzi A, Buda A, Belfiglio M, Valentini $\mathrm{M}$, et al. Efficacy of adjuvant chemotherapy after curative resection for gastric cancer: A meta-analysis of published randomised trials. A study of the GISCAD (Gruppo Italiano per lo Studio dei Carcinomi dell'Apparato Digerente). Ann.Oncol. 2000;11(7):837-43.

15. Janunger KG, Hafström L, Nygren P, Glimelius B. A systematic overview of chemotherapy effects in gastric cancer. Acta Oncol. 2001;40(2-3):309-26.

16. Sun P, Xiang JB, Chen ZY. Meta-analysis of adjuvant chemotherapy after radical surgery for advanced gastric cancer. Br.J.Surg. 2009;96(1):26-33.

17. Paoletti $\mathrm{X}$, Oba K, Burzykowski $\mathrm{T}$, Michiels $\mathrm{S}$, Ohashi Y, Pignon JP, et al. Benefit of adjuvant chemotherapy for resectable gastric cancer: A metaanalysis. JAMA 2010;303(17):1729-37.

18. Lee J, Lim DH, Kim S, Park SH, Park JO, Park YS, et al. Phase III trial comparing capecitabine plus cisplatin versus capecitabine plus cisplatin with concurrent capecitabine radiotherapy in completely resected gastric cancer with D2 lymph node dissection: The ARTIST trial. J.Clin. Oncol. 2012;30(3):268-73.

19. Bamias A, Karina M, Papakostas P, Kostopoulos I, Bobos M, Vourli G, et al. A randomized phase iii study of adjuvant platinum/docetaxel chemotherapy with or without radiation therapy in patients with gastric cancer. Cancer Chemother.Pharmacol. 2010; 65(6):1009-21. 
20. Yu C, Yu R, Zhu W, Song Y, Li T. Intensity-modulated radiotherapy combined with chemotherapy for the treatment of gastric cancer patients after standard D1/D2 surgery. J.Cancer Res.Clin.Oncol. 2012;138(2):255-9.

21. Fink U. Randomized phase III study of preoperative chemotherapy followed by surgery versus surgery alone in locally advanced gastric cancer. 1999. EORTC Protocol 40 954. European Organisation for Research and Treatment of Cancer, Brussels.

22. Dikken JL, van Sandick JW, Maurits Swellengrebel HA, Lind PA, Putter H, Jansen EPM, et al. Neo-adjuvant chemotherapy followed by surgery and chemotherapy or by surgery and chemoradiotherapy for patients with resectable gastric cancer (CRITICS). BMC Cancer 2011;11:Art. No. 329.

23. Ryoo BY, Kang YK, Min YJ, Zang DY, Kim GY, Yang DH, et al. Update of AMC 0201 study: A randomized phase III trial comparing mitomycin-C plus short-term doxifluridine (Mf) versus mitomycin-C plus long-term doxifluridine plus cisplatin (MFP) after curative resection of advanced gastric cancer (NCT00296335). J.Clin.Oncol. 2012;30(Suppl. 4):Abstr. 76.

24. Fuchs CS, Tepper JE, Niedzwiecki D, Hollis D, Mamon HJ, Swanson R, et al. Postoperative adjuvant chemoradiation for gastric or gastroesophageal junction (GEJ) adenocarcinoma using epirubicin, cisplatin, and infusional (CI) 5-FU (ECF) before and after CI 5-FU and radiotherapy (CRT) compared with bolus 5-FU/LV before and after CRT: Intergroup trial CALGB 80101. J.Clin. Oncol. 2011;29(Suppl.):Abstr. 4003.

25. Kang YK, Ryoo BY, Chang HM, Zang DY, Kim TW, Yang DH, et al. Update of AMC 0101 study: A phase III trial of intraperitoneal cisplatin and early mitomycin-C plus long-term doxifluridine plus cisplatin (iceMFP) versus mitomycin-C plus short-term doxifluridine (Mf) as adjuvant chemotherapy for grossly serosa-positive advanced gastric cancer (NCT00296322). J.Clin.Oncol. 2012;30(Suppl. 4):Abstr. 4.

26. Lauren P. The two histological main types of gastric carcinoma: Diffuse and so-called intestinal-type carcinoma. An attempt at a histo-clinical classification. Acta Pathol.Microbiol.Scand. 1965;64:31-49.

27. You WC, Blot WJ, Li JY, Chang YS, Jin ML, Kneller R, et al. Precancerous gastric lesions in a population at high risk of stomach cancer. Cancer Res. 1993; 53(6):1317-21.

28. Correa P, Haenszel W, Cuello C, Zavala D, Fontham E, Zarama $\mathrm{G}$, et al. Gastric precancerous process in a high risk population: Cross-sectional studies. Cancer Res. 1990;50(15):4731-6.

29. Peek Jr. RM, Blaser MJ. Helicobacter pylori and gastrointestinal tract adenocarcinomas. Nat.Rev.Cancer 2002;2(1):28-37.

30. Uemura N, Okamoto S, Yamamoto S, Matsumura N, Yamaguchi S, Yamakido M, et al. Helicobacter pylori infection and the development of gastric cancer. N.Engl.J.Med. 2001;345(11):784-9.

31. Carneiro F, Huntsman DG, Smyrk TC, Owen DA, Seruca R, Pharoah P, et al. Model of the early development of diffuse gastric cancer in E-cadherin mutation carriers and its implications for patient screening. J.Pathol. 2004;203(2):681-7.

32. Blot WJ, Devesa SS, Kneller RW, Fraumeni Jr. JF. Rising incidence of adenocarcinoma of the esophagus and gastric cardia. JAMA 1991;265(10):1287-9.

33. Crew KD, Neugut AI. Epidemiology of gastric cancer. World J.Gastroenterol. 2006;12(3):354-62.

34. Shah MA, Khanin R, Tang L, Janjigian YY, Klimstra DS, Gerdes $\mathrm{H}$, et al. Molecular classification of gastric cancer: A new paradigm. Clin.Cancer Res. 2011;17(9):2693-701. 